\title{
Toward Understanding the Interplay between Public and Private Healthcare Providers and Patients: An Agent-based Simulation Approach
}

\author{
Zainab Alalawi ${ }^{1}$, Yifeng Zeng ${ }^{2}$, The Anh $\operatorname{Han}^{1, *}$ \\ ${ }^{1}$ School of Computing, Engineering \& Digital Technologies, Teesside University, UK, \\ ${ }^{2}$ Department of Computer and Information Sciences, Northumbria University, UK
}

\section{Abstract}

Few modelling studies have been carried out to investigate patients' involvement in the decision-making process in a healthcare system. Here we perform theoretical and simulation analysis of a healthcare business model involving three populations: Public Healthcare Providers, Private Healthcare Providers and Patients. The analysis contributes to healthcare economic modelling by analyzing the dynamics and emergence of cooperative behavior of agents within the three populations. Resorting to agent-based simulations, we examine the effect of increasing behavioural mutation and providers' capacity on patients' cooperative behaviour. We show that the former introduces more randomness in agents' behaviors enabling cooperation to emerge in more difficult conditions. Moreover, when the providers' capacity to meet patients' demand is limited, patients exhibit low levels of cooperation, implying a more difficult cooperation dilemma in a healthcare system that needs addressing.

Received on 17 September 2020; accepted on 03 October 2020; published on 21 October 2020

Keywords: Healthcare Behaviour Modelling, Agent-based simulation, Evolutionary Game Theory, Collective Behaviour

Copyright ( $) 2020$ Zainab Alalawi et al., licensed to EAI. This is an open access article distributed under the terms of the Creative Commons Attribution license, which permits unlimited use, distribution and reproduction in any medium so long as the original work is properly cited.

doi:10.4108/eai.21-10-2020.166668

\section{Introduction}

The healthcare system in the UK is regulated by the Department of Health where explicit rules, activities and mechanisms are imposed and enforced by the state to manage social behavior [21]. From the perspective of UK health policy, the tendency is to understand an incentive as an economic inducement or benefit, whether tangible or intangible, resulting from a behavior that impacts the performance of the healthcare system, whether positively or negatively. This narrow definition tends to exclude non-monetary incentives or benefits [10, 21]. This issue can be solved pragmatically through the introduction of Evolutionary Game Theory (EGT) modelling [23], with various similar examples such as the Public Goods Game (PGG) and different forms of incentives (both monetary and non-monetary such as reputation-based ones) [18, 22, 24].

In this research, we investigate the evolution of cooperation in a complex system, namely the healthcare system in England, which is made up of populations

*Corresponding author. Email: t.han@tees.ac.uk consisting of different healthcare providers interacting with patients. Our investigation examines individual agents' behaviors as viewed by an external policymaker, in this case, the Health Department. Generally, policies are initiated and managed by the Health Department, which allocates a specific budget to interfere.

To this end, our analysis here is carried out based on a baseline model we developed in [2]. This previous work focuses on numerical analysis of the proposed healthcare model, studying evolutionary dynamics of three well-mixed finite populations. The analytical approach we adopted therein relied on the assumptions of rare behavioral mutation by the agents, and that all populations having an equal size. Our finding shows that agents from all three populations tend to not cooperate (i.e. defect) [2]. While these simplified assumptions allowed us, as a very first step, to provide clear mathematical analysis, they prevented us from analysing some important factors. Namely, mutation or behavioral exploration, where agents can freely experiment with new behaviors, has been shown to play an important role in enabling cooperation in the context of social dilemmas $[3,6,11,20,26]$. As the world 
is facing a rapid increase in populations, most healthcare capacity is struggling to cope with attendant mounting demand and to accommodate growing populations' needs. Moreover, some scholars have warned that, in reality, healthcare providers might not be able to meet the treatment demand from all patients [8]. It is, therefore, viable to investigate this intensifying trend and study the ratio of population to hospital bed availability. Currently in England, UK, the number stands at 474 patients per hospital bed ${ }^{1}$ [19].

Therefore, in this paper, we develop agent-based simulations of this baseline model to investigate how relaxing the above-mentioned assumptions affects the cooperation outcomes in providers' and patients' populations. Namely, we seek to develop a more insightful understanding of the dynamical behavior of agents in each population, the effects of applying a large mutation/exploration rate on the abundance of cooperation, and the influence of reduced sizes of the healthcare providers' population on the agent's behavior within the dynamic system and their population.

The rest of this paper is structured as follows. Related Work section reviews the most relevant literature. Model and Methods section presents our healthcare model and methods applied. Results section discusses agent-based simulation results. The conclusion summarises our findings and explores their implications for future work.

\section{Related Work}

Researchers eager to understand the behavior of different agent representations within the healthcare system use AI [30], game theory [1, 28], multiagents system [5] and big data [16] which are used to predict and understand behaviors within systems. However, little effort has been made to study the dynamics of cooperation and other decision making in the healthcare domain, which usually involves different actors in the decision making processes. Our previous work [2] and this work aim to bridge this important gap by resorting to population-based methods from EGT to develop an understanding of the dynamics of cooperative behaviour in this domain.

The rapid development in research on the learning of social behavior has significantly increased our understanding of the dynamic interaction among individuals from different populations [23]. Cooperation is one of the fundamental aspects to measure the strength and dynamism of a population $[7,14,25]$. It can be studied by applying EGT to different mechanisms, such as reciprocal behaviors, kin selection and costly incentives $[13,23]$. EGT has

\footnotetext{
${ }^{1}$ England population number was obtained from Office for National Statistic ONS (correct as of mid-2019), available at:https://bit.ly/2MCb3yc. The number of hospital beds in England (correct as of 2018/9) was calculated based on data provided by the King's fund report "NHS hospital bed numbers: past, present, future": https://bit.ly/2UfgeZ0; (accessed 6 June 2020).
}

\begin{tabular}{|l|c|}
\hline Parameters' description & Symbol \\
\hline \hline $\begin{array}{l}\text { Reputation benefit for the Public and } \\
\text { Private healthcare providers }\end{array}$ & $b_{R}$ \\
\hline Patient's benefit & $b_{P}$ \\
\hline $\begin{array}{l}\text { Cost of investment spent by the Pub- } \\
\text { lic/Private healthcare provider }\end{array}$ & $c_{I}$ \\
\hline $\begin{array}{l}\text { Cost of treatment acquired by the } \\
\text { healthcare provider }\end{array}$ & $c_{T}$ \\
\hline Cost of healthcare management & $c_{M}$ \\
\hline $\begin{array}{l}\text { Extra Patient's benefit when both } \\
\text { providers cooperate }\end{array}$ & $\varepsilon$ \\
\hline
\end{tabular}

\begin{tabular}{|l|l|l||l|l|l|}
\hline \multicolumn{2}{|l|}{ Strategies } & \multicolumn{4}{l|}{ Payoffs } \\
\hline P1 & P2 & P3 & Public & Private & Patient \\
\hline \hline$C$ & $C$ & $C$ & $\begin{array}{l}b_{R}-c_{I}- \\
c_{T}\end{array}$ & $\begin{array}{l}b_{R}-c_{I}- \\
c_{M}\end{array}$ & $b_{P}+\varepsilon b_{P}$ \\
\hline$C$ & $C$ & $D$ & $-c_{I}$ & $-c_{I}$ & 0 \\
\hline$C$ & $D$ & $C$ & $\begin{array}{l}b_{R}-c_{I}- \\
c_{T}\end{array}$ & 0 & $b_{P}$ \\
\hline$C$ & $D$ & $D$ & $-c_{I}$ & 0 & 0 \\
\hline$D$ & $C$ & $C$ & 0 & $\begin{array}{l}b_{R}-c_{I}- \\
c_{M}\end{array}$ & $b_{P}-c_{T}$ \\
\hline$D$ & $C$ & $D$ & 0 & $-c_{I}$ & 0 \\
\hline$D$ & $D$ & $C$ & 0 & 0 & $-c_{T}$ \\
\hline$D$ & $D$ & $D$ & 0 & 0 & 0 \\
\hline
\end{tabular}

Table 1. The healthcare model (Public: P1, Private: P2, Patient: P3).

been successfully used across major scientific fields such as biology, ecology, economics, psychology and mathematical computation $[13,23]$.

\section{Model and Methods}

\subsection{Healthcare Model}

In this section, we summarise the model presented in [2], where we consider three populations: Public providers $P 1$, Private providers $P 2$ and Patients $P 3$. While $P 1$ represents the NHS or the public healthcare providers, $P 2$ represents independent healthcare providers $P 2$ selling healthcare services, and $P 3$ represents those who seek personal treatments. Agents from each population $P 1, P 2$ and $P 3$ can choose from two strategies: provide/accept sustainable treatment identified as cooperating, otherwise the game will be dominated by alternative treatments from other providers. On each game encounter or iteration, an agent's payoff is acquired based on the strategy played by each agent/individual from the three populations. In this game, an interaction happens among three agents, one from each of the three populations, see Table 1.

An agent in each of the three populations has two strategies, cooperate $(C)$ and defect $(D)$. Here the strategy coincides with the action of an agent, despite the fact that there is only one decision to make, which can be interpreted 
as follows.

\section{Public healthcare providers:}

- $C$ : offers treatment paid for from taxpayers' money, which consists of paying a $\cos t c_{I}$, and in return gets a reputation benefit.

- $D$ : does not want to pay for the treatment.

\section{Private healthcare providers:}

- $C$ : offers treatment either paid by Public (when Public cooperates) or self-paid by Patient (so the main cost involved is management cost $c_{M}$ ), and obtains a reputation benefit $b_{R}$. In case of cooperation with the Patient, Private commits to investing $c_{I}$ from its revenue.

- $D$ : does not want to offer the treatment.

\section{Patient:}

- $C$ : accepts being treated and pays for the treatment $c_{T}$ when treated by the Private; Patient obtains health benefit $b_{P}$ if at least one provider cooperates and extra health benefit when both providers cooperate $\varepsilon$.

- $D$ : rejects the treatment and looks for alternative treatment mostly overseas, where above conditions are correct, and: $b_{P}>0, \varepsilon>0$ and $c_{T}>0$.

Thus, given the strategies of players in each population, there are 8 possible strategic combinations or scenarios, represented by XYZ where X, Y, Z can be either C or D. We summarize a few of them below (full details can be found in [2])

- $C C C$ : individuals from all three populations choose to cooperate. The Public pays for the treatment provided by a Private healthcare provider and the Patient accepts the provided treatment in pursuit of her/his own benefit or well-being. The Public, which bears the costs of investment and pays for the Patient's treatment $\left(c_{I}+c_{T}\right)$, covered from its allocated budget, gains a reputation benefit $b_{R}$. Reputation benefits are derived from the Patient's satisfaction with the provided service. On the other hand, the Private healthcare provider will provide the required treatment to the Patient and receive the payment covering the costs from the Public. The cost of investment $c_{I}$ of the Private healthcare provider is to invest in staffing and healthcare facilities, while $c_{M}$ refers to administrative and operational costs. Also, the Private provider will obtain a reputation benefit with the Public $b_{R}$. Patient gets extra health benefit $\varepsilon b_{P}$ as both healthcare providers are cooperating ( $\varepsilon$ represents a synergistic factor).

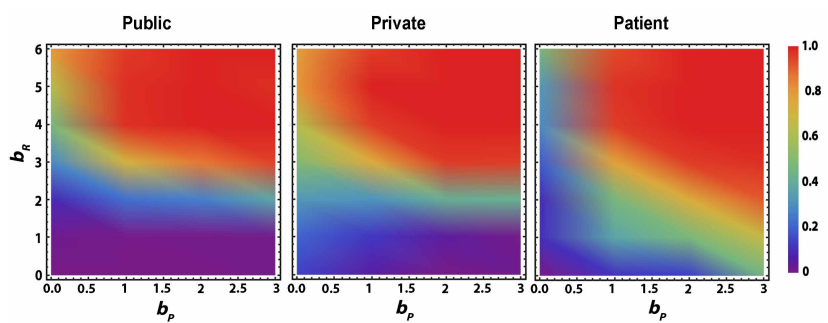

Figure 1. The cooperation level in each of the three populations as a function of the sectors' benefit $b_{R}$ and patient's benefit $b_{P}$. Other parameters: $c_{I}, c_{T}, c_{M}=$ $1 ; N_{1}, N_{2}, N_{3}=100 ; \varepsilon=0.2 ; \mu=10^{-4} ; \beta=0.1$.

- $D D D$ : this scenario consists of all three agents choosing not to interact with one another, thus all the agents get a zero payoff.

\subsection{Method: Evolutionary Dynamics for Three Populations}

EGT method is adopted to study the evolutionary dynamics and interactions among individuals from three distinct finite populations: $P 1, P 2$ and $P 3$. The populations are of fixed sizes $N_{1}, N_{2}$ and $N_{3}$, respectively. In each time step, from a randomly selected population, a randomly selected individual $A$ with fitness $f_{A}$ imitates another randomly selected individual $B$ with $f_{B}$ fitness using the pairwise comparison rule, a popular and standard approach to implementing social learning in EGT [23, 27]. Namely, the probability $\rho$ that A adopts B's strategy is given by the Fermi's function

$$
\rho=\left[1+e^{-\beta\left[f_{B}-f_{A}\right]}\right]^{-1},
$$

where $\beta>0$ represents the 'intensity of selection' or 'imitation strength'; $\beta=0$ represents neutral drift where imitation decision is random, while for $\beta \rightarrow \infty$ the imitation decision is increasingly deterministic. In our results, we set $\beta=0.1$, which is in accordance with previous theoretical and experimental works [20, 29].

When mutation or behavioral exploration $[6,9,20]$ is not rare, we consider that, before $\mathrm{A}$ considers to imitate $\mathrm{B}$, with a probability $\mu$, it changes to a randomly selected strategy from its behavioural space (in this case, C or D). That is, with probability $(1-\mu)$ A considers to imitate $\mathrm{B}$ as above.

Individuals in each population have the choice to play $C$ or $D$. As described above, there are eight possible scenarios corresponding to the the eight possible combinations of the strategies within the three populations: $C C C, C C D, C D C$, $C D D, D C C, D C D, D D C, D D D$. Denoting the numbers of cooperators in $P 1, P 2$ and $P 3$ by $x, y$, and $z$, respectively, the payoff of each strategy can be written as follows:

$$
\left.\begin{array}{rll}
P_{S}^{\text {Public }}(x, y, z) & = & P_{\text {syz }} \\
P_{S}^{\text {Private }}(x, y, z) & = & P_{x s z} \\
P_{S}^{\text {Patient }}(x, y, z) & = & P_{x y s}
\end{array}\right\} \quad \text { Pairwise comparison }
$$




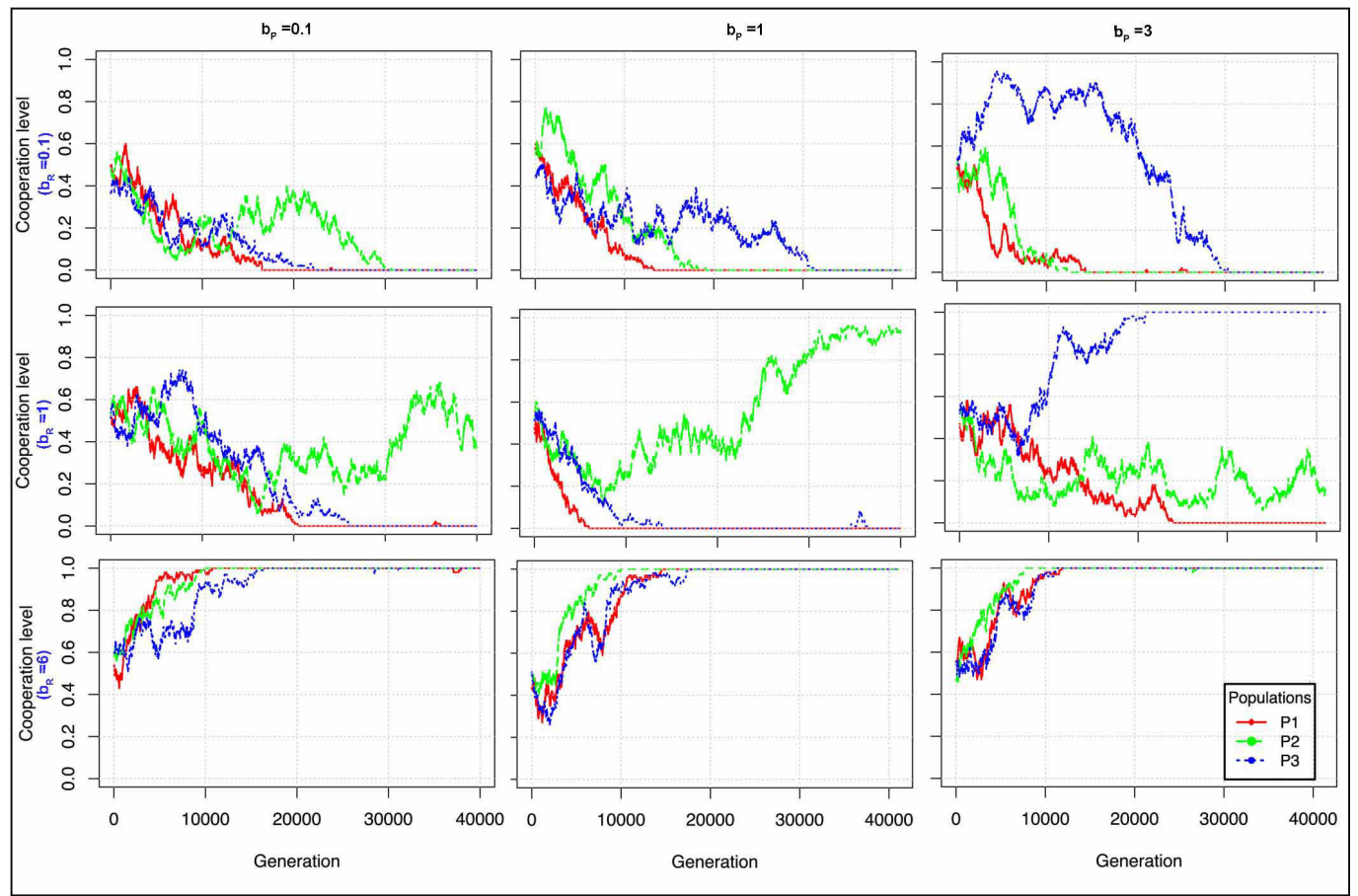

Figure 2. Typical simulation runs showing the cooperation levels over time in the three populations (red for Public, blue for Private and green for Patient). The plots represent the frequency of cooperation adopted by the populations for different values of $b_{R}$ and $b_{P}$. Other parameters as in Figure 1.

where $P_{x y z}$ is the payoff for the strategy selected by individuals from one of the stated populations, and $(x, y, z)$ represents the selected strategies $C$ or $D$. For instance, individuals from $P l$ have the options to play $C$ or $D$. The selected strategy will replace the $s$ at $x$ vertex, while $y$ and $z$ vertices remain unchanged for selected strategies for the Public population in this instance.

$$
\begin{aligned}
& \text { Public } \rightarrow f(C y z)>P_{D y z} \\
& \text { Private } \rightarrow f(x C z)>P_{x D z} \\
& \text { Patient } \rightarrow f(x y C)>P_{x y D}
\end{aligned}
$$

\subsection{Agent-based Simulations}

As mutation is not rare, we perform agent-based simulations to study the evolutionary dynamics. Initially, agents in the three populations are given a random strategy $(C$ or $D)$. In each generation or time step, agents' fitness is calculated as in Table. 1. Namely, the fitness of an individual adopting a strategy $s$ within a population is derived from the average obtained from the tripartite oneshot game described in Table 1. A randomly selected individual in each game obtains an average payoff given by $[7,22]$ :

$$
\begin{aligned}
& f_{S}^{\text {Public }}(x, y, z)=y z P_{S C C}^{\text {Public }}+(1-y) z P_{S D C}^{\text {Public }}+y(1- \\
& z) P_{S C D}^{\text {Public }}+(1-y)(1-z) P_{S D D}^{\text {Public }}, \\
& f_{S}^{\text {Private }}(x, y, z)=x z P_{C S C} \text { Private }+(1-x) z P_{D S C}^{\text {Private }}+ \\
& x(1-z) P_{C S D}^{\text {Private }}+(1-s)(1-z) P_{D S D}^{\text {Private }},
\end{aligned}
$$

$$
\begin{aligned}
& f_{S}^{\text {Patient }}(x, y, z)=x y P_{C C S}^{\text {Patient }}+(1-x) y P_{D C S}^{\text {Patient }}+x(1- \\
& \text { y) } P_{C D S}^{\text {Patients }}+(1-x)(1-y) P_{D D S}^{\text {Patient }},
\end{aligned}
$$

where $f_{S}^{P}(x, y, z)$ represents agents' average payoff in a population $P$ while adopting strategy s, assuming that the population is present in the vertices $(\mathrm{x}, \mathrm{y}, \mathrm{z}) . P_{A B C}^{S}$ denotes the payoff that an individual playing in a group with a strategy profile derives from state $\mathrm{S}$ where public plays $\mathrm{A}$, private plays $\mathrm{B}$ and patient plays $\mathrm{C}$. These payoffs are shown in Table 1.

Each simulation of the stochastic modelling runs for a number of generations (at least 40,000), where a stable state is reached in general (see examples in Fig. 2). To ensure accuracy, the accumulated results are then averaged over the last 100 time steps, and furthermore, for each parameter configuration, the results were averaged over 50 independent realisations. As mutation is adopted in the simulations, no absorbed monomorphic state is reached .

\section{Results}

In this study we conduct agent-based simulations (see Methods), where agents from the three populations $P 1, P 2$ and $P 3$ interact in a one-shot game and learn how they influence the level of cooperation to sustain cost-effective services and better patient satisfaction.

Figure 1 shows the cooperation levels adopted in the populations for varying the sectors' benefit $b_{R}$ and patient's 
benefit $b_{P}$. In general, cooperation in all populations is most abundant when both benefits are sufficiently large. The two sectors have similar cooperation for the whole parameter space, except when both benefits are small, where the private sector has a slightly higher level of cooperation.

To better understand the detailed dynamics, in Figure 2 we show the evolution of cooperation over time in each population for different combinations of the benefits. Indeed, we can see that when $b_{R}$ is large (see $b_{R}=6$, bottom row), all the three populations quickly converge to full cooperation even for small $b_{P}$. For intermediate $b_{R}$ (see $b_{R}=1$, middle row), $P 1$ converges to defection, while $P 2$ still maintains some level of cooperation and $P 3$ have high levels of cooperation when $b_{P}$ is large (i.e. equals 3 ). When $b_{R}$ is small, all populations converge to defection. That is, $P 2$ population is more willing to cooperate with $P 3$ for quite low $b_{P}$ (where $b_{P} \leq 1$ and $\left.b_{R} \leq 1\right)$. On the other hand, $P 3$ requires better reputation benefit $b_{R}$ to cooperate with $P 3$ and provides the desired services. As a consequence, $P 3$ has to pay extra cost equivalent to $c_{T}$ as represented in Table 1 , i.e: $D C C$ for the Patient's payoff $\left(b_{P}-c_{T}\right)$ compared to the other scenarios, $C C C$ or $C D C$, where the cost equals zero.

\subsection{The effect of mutation}

We now study how increasing the mutation rate $\mu$ affects the cooperation outcome in each population. Note that so far we consider rare mutation or low mutation rate $\mu=$ $10^{-4}$, recovering results from our previous work using small mutation dynamics [2]. Fig. 4 shows results for higher mutation rates, namely, $\mu=10^{-k}$, with $k=1.5,1$ and 0.5. As expected, as larger mutation leads to greater levels of randomness in agents' behaviors, we observe more defection in scenarios where cooperation is abundant (high benefits), and vice versa, i.e. more cooperation in
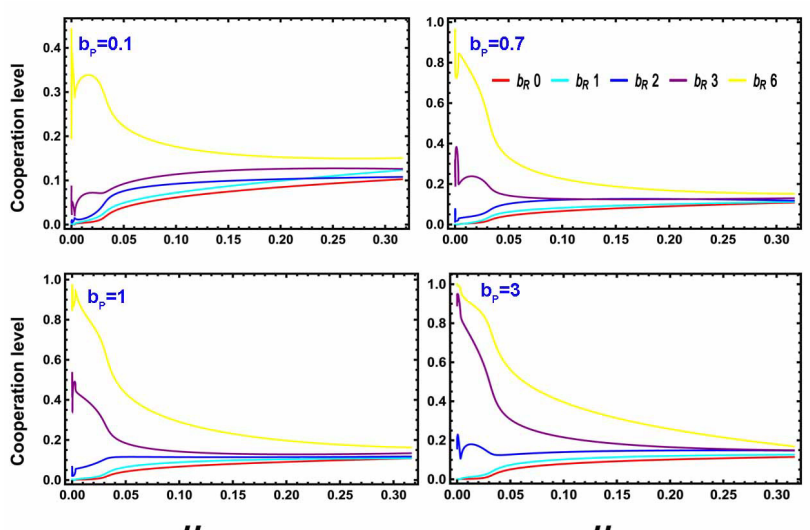

$\boldsymbol{\mu}$

Figure 3. Frequencies of $C C C$ for increasing mutation rate, $\mu$. It represents the trends of different $b_{R}$ values for the stated value of $b_{P}$ on each plot. Other parameters as in Figure 1.
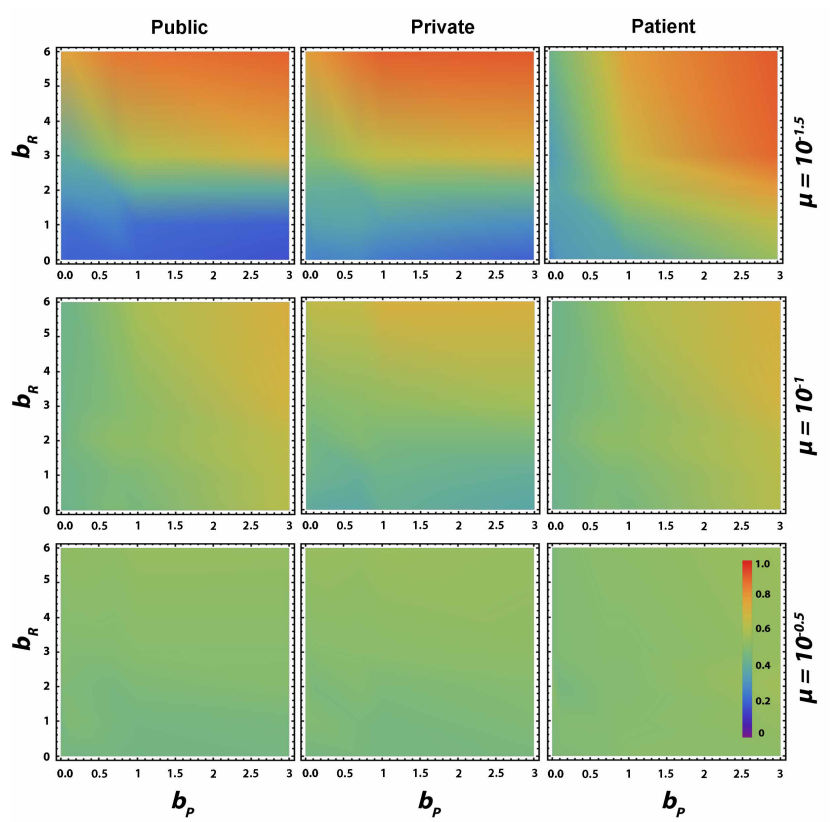

Figure 4. Cooperation levels in three populations for different values of $\mu$. First column for $P 1$, second column for P2, and third column for P3. $\mu$ values are as specified on the right side. Other parameters as in Figure 1.

previous scenarios with abundant defections. Interestingly, for intermediate $\mu\left(\mu=10^{-1}\right), P$ l's cooperation mostly depends on $P 3$ 's benefit, while $P 2$ is more dependent on the reputation benefit. This observation can also be seen in Fig. 3, where we plot the frequency of $C C C$. For sufficiently large $\mu$, the frequency of $C C C$ always converges to $1 / 8$ regardless of the values of the benefits, which is when all populations converge to $50 \%$ cooperation.

\subsection{The effect of unequal population sizes}

In reality, the availability of hospital beds is very limited for the increasing number of patients; i.e: winter long waiting time in A\&E or in a pandemic (e.g. as is the case with the COVID-19). We study the effect of this factor by looking at different providers' population size ratios to that of the Patient's population. Fig. 5 shows simulation results for two different providers' sizes, $10 \& 5$. The results reveal that $P 2$ cooperates more with lower providers' and patients' benefits. Whereas the $P 1$ population still prefers to cooperate with higher reputation benefit $b_{R}$. Conversely, $P 3$ shows a significantly different behavior in the decision making process by defecting upon low health benefit $0.1<$ $b_{P}<1$ and willing to cooperate when $b_{P}<0.1$ and $b_{P} \geq 1$ for larger providers' population sizes.

These observations show that, in a more realistic setting where both $P 1$ and $P 2$ usually have a lower capacity than what is required by $P 3$, it is more difficult to ensure 

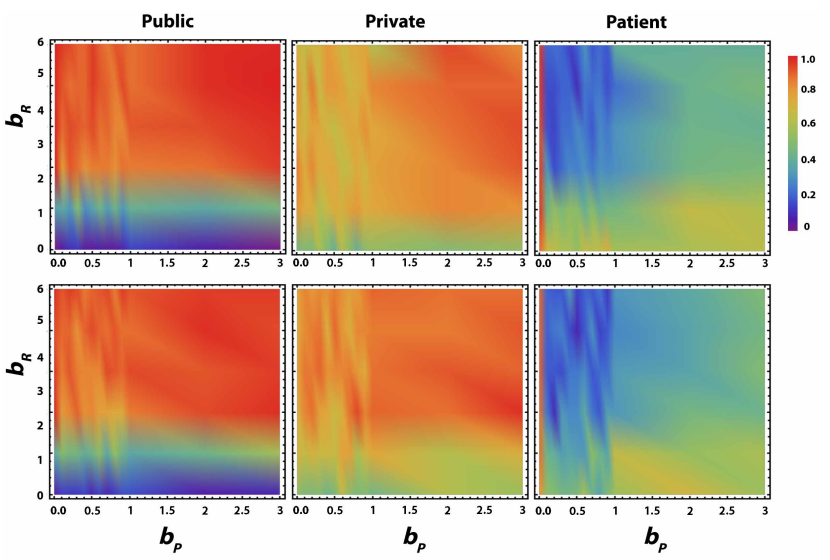

Figure 5. Cooperation levels in each population based on the ratio of hospital bed availability provided by healthcare providers compared to the size of the $P 3$ population $\left(N_{3}=100\right)$. Where the size of the $P 1, P 2$ is: first row $N_{1}=N_{2}=10$ and second row $N_{1}=N_{2}=5$. Other parameters as in Figure 1.

cooperation from the patients. That is, the cooperation issue is more severe, which therefore requires additional supporting mechanisms such as incentives to maintain cooperation. We are currently studying this important setting.

\section{Conclusions}

We have studied cooperation outcomes and evolutionary dynamics of a three-population healthcare model using extensive agent-based simulations. We analyze cooperation outcomes for incremental rate of mutation, and compare them with those when it is rare, as reported in our previous work. This result can represent realistic scenarios derived from some elective treatments such as hip replacements [15]. To link our model to realistic scenarios it will make use of data published by the NHS and complemented with figures obtained from the Patient Reports Outcome Measures [17] and the Care Quality Commission [4].

We found abundance of cooperation closely linked with high benefits. Interestingly, for sufficiently high mutation rates, more cooperation also emerged in scenarios that are previously dominated by defection in all populations.

We explored the effect of reducing the size of the healthcare provider population on cooperation between the three populations. We found that the Patient is less likely to cooperate (small abundance) when the Providers' populations are small, which means that providers have a limited capacity to accommodate the patients' needs. This finding reveals a critical cooperation problem that needs to be resolved. This is an important issue since in reality providers might not always be able to meet patients' demand, especially when health crises occur such as in the time of a pandemic (such as COVID-19). Again, our findings reiterate the important role of patients in healthcare decision making processes, which has been pointed out in previous reports [17] but usually omitted in modelling works.

As future work, we plan to explore new mechanisms to improve cooperation in this important and realistic setting, such as via positive or negative incentives in the form of peer and institutional rewards and punishments $[2,12,24]$. Moreover, in this paper, as a first step, we assumed that providers have the same capacity. However, in reality, the public and private sectors may have very different capacities, which might depend on different regions and their local demands. We plan to provide a more comprehensive analysis of how difference in providers' capacities, in relation to the patients' demand [19].

\section{Acknowledgment}

YZ is supported by EPSRC project No. (EP/S011609/1). TAH is supported by Future of Life Institute (grant RFP2154).

\section{References}

[1] Agee, M. D. and Gates, Z. (2013). Lessons from game theory about healthcare system price inflation. Applied health economics and health policy, 11(1):45-51.

[2] Alalawi, Z., Han, T. A., Zeng, Y., and Elragig, A. (2019). Pathways to good healthcare services and patient satisfaction: An evolutionary game theoretical approach. In The 2019 Conference on Artificial Life (ALIFE), pages 135-142. MIT Press.

[3] Antal, T., Traulsen, A., Ohtsuki, H., Tarnita, C. E., and Nowak, M. A. (2009). Mutation-selection equilibrium in games with multiple strategies. Journal of theoretical biology, 258(4):614-622.

[4] CQC (2019). Care Quality Commission for All Healthcare services in the UK. https://bit.ly/2VkFKyx.

[5] De, Soham; Nau, D. S. and Gelfand, M. J. (2017). Understanding norm change: An evolutionary game-theoretic approach. In Proceedings of the 16th Conference on Autonomous Agents and MultiAgent Systems, pages 14331441. International Foundation for Autonomous Agents and Multiagent Systems.

[6] Duong, M. H. and Han, T. A. (2019). On equilibrium properties of the replicator-mutator equation in deterministic and random games. Dynamic Games and Applications, pages $1-23$.

[7] Encarnação, S., Santos, F. P., Santos, F. C., Blass, V., Pacheco, J. M., and Portugali, J. (2016). Paradigm shifts and the interplay between state, business and civil sectors. Royal Society Open Science, 3(12):160753.

[8] Ewbank, L., Thompson, J., and McKenna, H, a. S. A. (2020). NHS hospital bed numbers: past, present, future. the Kings Fund.

[9] Gokhale, C. S. and Traulsen, A. (2010). Evolutionary games in the multiverse. Proceedings of the National Academy of Sciences, 107(12):5500-5504. 
[10] Grant, J. (2003). Incentives for Reform in the NHS: An assessment of current incentives in the south-east London health economy - Jenny Grant - The King's Fund, 29th September 2005. Technical report, The king's fund.

[11] Han, T. A., Pereira, L. M., and Santos, F. C. (2012). The emergence of commitments and cooperation. In Proceedings of the 11th International Conference on Autonomous Agents and Multiagent Systems (AAMAS'2012), pages 559-566. ACM.

[12] Han, T. A. and Tran-Thanh, L. (2018). Cost-effective external interference for promoting the evolution of cooperation. Scientific reports, 8(1):1-9.

[13] Hofbauer, J. and Sigmund, K. (1998). Evolutionary games and population dynamics. Cambridge university press.

[14] Kurokawa, S. and Ihara, Y. (2009). Emergence of cooperation in public goods games. Proceedings of the Royal Society of London B: Biological Sciences, 276(1660):13791384.

[15] Moscelli, G., Gravelle, H., Siciliani, L., et al. (2016). Market structure, patient choice and hospital quality for elective patients. Technical report, Centre for Health Economics, University of York.

[16] Murdoch, T. B. and Detsky, A. S. (2013). The inevitable application of big data to health care. Jama, 309(13):13511352.

[17] NHSDigital (2019). Finalised Patient Reported Outcome Measures (proms) in England for Hip and Knee Replacement Procedures (April 2017 to March 2018). https://bit.ly/2J3Rq2h.

[18] Nowak, M. A., Sasaki, A., Taylor, C., and Fudenberg, D. (2004). Emergence of cooperation and evolutionary stability in finite populations. Nature, 428(6983):646.

[19] Office for National Statistic (2020). Population estimates for the UK, England and Wales, Scotland and Northern Ireland, provisional: mid-2019.

[20] Rand, D. G., Tarnita, C. E., Ohtsuki, H., and Nowak, M. A. (2013). Evolution of fairness in the one-shot anonymous ultimatum game. Proceedings of the National Academy of Sciences, 110(7):2581-2586.
[21] Saltman, R. B. (2002). Regulating incentives: the past and present role of the state in health care systems. Social Science \& Medicine, 54(11):1677-1684.

[22] Santos, F. P., Encarnação, S., Santos, F. C., Portugali, J., and Pacheco, J. M. (2016). An evolutionary game theoretic approach to multi-sector coordination and self-organization. Entropy, 18(4):152.

[23] Sigmund, K. (2010). The calculus of selfishness. Princeton University Press.

[24] Sigmund, K., De Silva, H., Traulsen, A., and Hauert, C. (2010). Social learning promotes institutions for governing the commons. Nature, 466(7308):861.

[25] Smith, J. M. (1974). The theory of games and the evolution of animal conflicts. Journal of theoretical biology, 47(1):209221.

[26] Traulsen, A., Hauert, C., De Silva, H., Nowak, M. A., and Sigmund, K. (2009). Exploration dynamics in evolutionary games. Proceedings of the National Academy of Sciences, 106(3):709-712.

[27] Traulsen, A., Pacheco, J. M., and Nowak, M. A. (2007). Pairwise comparison and selection temperature in evolutionary game dynamics. Journal of theoretical biology, 246(3):522529 .

[28] Wu, C.-K., Chen, Y.-M., and Wu, D. (2016). A game theory approach for deploying medical resources in emergency department. In Game Theory and Applications, pages 18-30. Springer.

[29] Zisis, I., Di Guida, S., Han, T., Kirchsteiger, G., and Lenaerts, T. (2015). Generosity motivated by acceptanceevolutionary analysis of an anticipation game. Scientific reports, 5(1):1-11.

[30] Ziuziański, P., Furmankiewicz, M., and SołtysikPiorunkiewicz, A. (2014). E-health artificial intelligence system implementation: case study of knowledge management dashboard of epidemiological data in poland. International Journal of Biology and Biomedical Engineering, 8:164-171. 